\title{
Representation of European hydroclimatic patterns with Self-Organizing
}

\author{
Maps \\ Yannis Markonis* and Filip Strnad \\ Faculty of Environmental Sciences, Czech University of Life Sciences Prague, Kamycka 129, \\ Praha - Suchdol, 165 00, Czech Republic
}

${ }_{6}^{*}$ Corresponding author address: Yannis Markonis, Faculty of Environmental Sciences, Czech Uni-

7 versity of Life Sciences Prague, Kamycka 129, Praha - Suchdol, 165 00, Czech Republic.

s E-mail: markonis@fzp.czu.cz 


\begin{abstract}
9 Self-Organizing Maps provide a powerful, non-linear technique of dimen10 sionality reduction that can be used to identify clusters with similar attributes. ${ }_{11}$ Here, they were constructed from a 1000-year-long gridded palaeoclimatic 12 dataset, namely the Old World Drought Atlas, to detect regions of homoge13 neous hydroclimatic variability across the European continent. A classifica14 tion scheme of 10 regions was found to describe most efficiently the spa15 tial properties of Europe's hydroclimate. These regions were mainly divided ${ }_{16}$ into a northern and a southern subset, linked together with a northwest-to${ }_{17}$ Southeast orientation. Further analysis of the classification scheme with com${ }_{18}$ plex networks confirmed the divergence between the northern and southern 19 components of European hydroclimate, also revealing that is not strongly cor${ }_{20}$ related to the Iberian peninsula. On the contrary, the region covering British ${ }_{21}$ Isles, France and Germany, appeared to be linked to both branches, implying 22 links of hydroclimate with atmospheric/oceanic circulation.
\end{abstract}




\section{Introduction}

In the last decades, the available amount of data in earth sciences has been exponentially increasing. The explosion of data includes numerous different sources and technologies, spanning from high-resolution satellite data to multi-proxy reconstructions of past hydroclimates. At the same time, there has been a significant progress in empirical data-driven techniques, widely known as machine learning. This is because while the collection of large volumes of data is essential in almost every field of geosciences, analyzing this information becomes more challenging. Evidently, the magnitude of such big datasets has prominent effects both to the information extraction and interpretation methods. Traditional analysis approaches are not suitable to investigate or utilize such massive data products. Hence, alternatives revolving around machine learning methods that can be implemented for forecasting or classification are becoming increasingly popular (Lary et al. 2016; Papacharalampous et al. 2017; Tyralis et al. 2019).

Machine learning techniques have not been unknown in paleoclimatology. Their first applications can be mainly found in paleoceanography (Malmgren and Nordlund 1997; Pozzi et al. 2000; Peyron and Vernal 2001; Cortese et al. 2005) and dendroclimatology (Keller et al. 1997; Woodhouse 1999; D'Odorico et al. 2000; Carrer and Urbinati 2001; Ni et al. 2002). In all these studies, artificial neural networks were used to calibrate the relationship between the relationship between the observational records and proxy time series for an overlapping period. Thus, artificial neural networks were found to be plausible alternatives to transfer functions for temperature reconstructions. Additionally, they were also used for interpolation of paleovegation data at global scale (Grieger 2002), as well as in multi-proxy reconstructions (Guiot et al. 2005). Artificial neural networks still remain popular today (Carro-Calvo et al. 2013; Pérez-Ortiz et al. 2019), while other promising approaches, such as boosted regression trees, are also utilized (Salonen et al. 2014; Jug- 
gins et al. 2015). We can see that the main application of machine learning in paleoclimatology is for transforming the climate signal encapsulated to proxy time series to climate variables.

On the other hand, machine learning techniques for classification are quite uncommon in paleoclimatic studies. Only recently, there were a few developments in this field. If we exclude some specialized applications, e.g., rain-to-grain classification of pollen data (Punyasena et al. 2012), the main use is of detection of spatial patterns in gridded paleoclimatic reconstructions with SelfOrganizing Maps (SOMs) (Reusch 2010; Wise and Dannenberg 2014; Edwards et al. 2017). SOMs have been widely applied in climatology and hydrology, including regional frequency analysis of precipitation Lin and Chen (2006), assessment of the variability of daily evaporation (Chang et al. 2010), investigation of precipitation characteristics (Hsu and Li 2010), circulation patterns (Cavazos 2000; Cavazos et al. 2002; Rousi et al. 2017), catchment classification (Ley et al. 2011; Prinzio et al. 2011; Toth 2013; Farsadnia et al. 2014), pre-processing of precipitation satellite data (Nourani et al. 2013), station classification for drought determination (Rad and Khalili 2015), hydroclimatic variable classification for water management (Rodríguez-Alarcón and Lozano 2017) and investigation of long-term persistence in streamflow (Markonis et al. 2018b). The increasing number of studies adopting SOMs, highlights their potential and efficiency, in the investigation of spatiotemporal properties of gridded paleoclimatic records.

In this study, we apply Self-Organizing Maps, to study the spatial patterns in Europe's hydroclimate during the last one thousand years. Our aim is not only to detect the areas with substantial homogeneity during European droughts and pluvials, but also provide a comprehensive demonstration of this data-driven classification method. This is complemented with a freely available software application, namely the somspace $\mathrm{R}$ package. In this manner, we hope that will further support spatiotemporal analyses in reconstructed datasets, which are being increasingly important in paleoclimatic studies. 


\section{Material \& Methods}

Paleoclimatic reconstructions of hydroclimatic variables have been introduced into hydrology to describe streamflow (Schook et al. 2016; Ho et al. 2016, 2017), floods (Benito et al. 2004), average (Ho et al. 2015a,b) or extreme rainfall (Steinschneider et al. 2016) and drought (Cook et al. 2004, 2015). Except for a number of regional studies, e.g., for British Isles (Spraggs et al. 2015) or France (Caillouet et al. 2017), reconstructions often focus on meteorological drought. However, the impacts of hydrological drought are more heterogeneous in space and time than those of meteorological drought being linked significantly to hydrological preconditions, which have to be known to assess the development of hydrological drought from meteorological drought as well as its impacts on water resources.

A prominent case is the Old World Drought Atlas (OWDA), a tree-ring reconstruction of the self-calibrated Palmer Drought Severity Index (scPDSI) over Europe and parts of Northern Africa and Middle East for the last 2,000 summers (Cook et al. 2015). The OWDA follows the methodology applied in similar studies about long-term drought behaviour over North America (Cook et al. 2004) or Asia (Cook et al. 2010). Since its release it has already been used to decipher European hydroclimatic multi-decadal variability (Markonis et al. 2018a), determine the magnitude of Mediterranean drying (Cook et al. 2016) and to investigate the tele-connection signals in temperature and precipitation across Northern Hemisphere (Baek et al. 2017).

The OWDA has been compiled by the spatial regression of 106 tree-ring chronologies to a map with 5414 half-degree grid cells at a $0.5 \times 0.5$ resolution. Here, we use the data subset extracted by Markonis et al. (2018a), which optimizes the temporal and spatial distribution of the dataset, following two criteria: (a) the grid cell reconstructions are based on at least 20 tree-ring chronologies within a $1000 \mathrm{~km}$ radius, as indicated by Cook et al. (2015) and (b) all reconstructions 
have tree-ring chronologies of similar length. The resulting data grid covers $35.25 \mathrm{~N}-62.75 \mathrm{~N}$ and 4.25W - 36.25E (1940 grid cells) for the period 992 - 2012 AD. We should note that although the scPDSI is reconstructed for the summer season, i.e., a single mean value for JJA, it has been demonstrated to be strongly correlated with annual scPDSI (Markonis et al. 2018a).

The methodological framework applied was based on the Self-Organizing Map (SOM) algorithm. SOM is an iterative process, which transforms the original dataset to a smaller representative set of nodes. The resulting subset is usually presented through a two-dimensional output layer (unified-distance matrix or U-Matrix), where each node corresponds to a group of members of the original dataset that share some features as determined by some distance measure (Ultsch and Siemon (1990)). In addition, the positioning of the nodes in the output layer presents their (non-linear) relationships, as nodes that are closer are more similar. This allows for enlightening visualizations of the data space,by presenting clusters with similar properties and their interdependencies. For readers interested in the specifics of the algorithm and its properties, we would recommend the work of Kohonen (2001), while a review of the SOM approach in water resources has been presented by Kalteh et al. (2008).

An advantage of the method is that the number of classes neither their range is not determined a priory, but results from the process itself. The number of nodes of the SOM is predefined though, with no single method for its determination. The most common practice is based on the comparison of differently sized SOMs and the selection of the one that minimizes homogeneity measure, while at the same time preserving noticeable levels of clustering and offers a substantial comprehensibility (Chang et al. 2010; Ley et al. 2011; Rousi et al. 2017). To achieve this one can either select a number of nodes which will represent the final classification scheme or construct a SOM with more nodes than the expected number of clusters and then apply some secondary classification technique to the output layer (two-layer SOM). The first approach can be used when 
the number of datasets is small and/or there is some preliminary evidence about the number of clusters that describe the data efficiently (Prinzio et al. 2011; Toth 2013). However, this is obviously subjective and in the case of larger datasets it is likely to overestimate the number of clusters (Hsu and Li 2010). The two-layer SOM approach allows a more detailed investigation of potential classification regimes and has been found to present more explicit results (Vesanto and Alhoniemi 2000).

In the two-layer SOM case, it is generally agreed that although U-matrix is an efficient first approach to visually inspect the number of clusters, it should not be used to determine the cluster boundaries on resulting 2d lattice and form the final clusters Farsadnia et al. (2014). Hence numerous methods have been applied to subdivide the output layer, including hierarchical agglomerative clustering using the Wards method (Hentati et al. 2010), partition clustering using the k-means method (Vesanto and Alhoniemi 2000) and the fuzzy clustering method (Srinivas et al. 2008; Giraudel et al. 2000). In addition, it has also been suggested to apply a second smaller SOM for cluster detection with promising results (Hsu and Li 2010; Nourani et al. 2013). Since each classification method has its own strengths and shortcomings, they should always be used with caution. If the resulting clusters are unclear or incomprehensible, then it could be useful to compare different classification algorithms, against validation measure(s).

Such measures are also appropriate for the determination of the representative number of clusters. The main principle is that the variance within each cluster should be minimized, whereas the variance between clusters should be maximal. Such criteria include the the $\mathrm{CH}$ index (Caliński and Harabasz 1974), C-index (Hubert and Schultz 1976) and the DB index (Davies and Bouldin 1979). A more hydrological-centered test for regional homogeneity based on the L-moments theory was developed by Hosking and Wallis (1997) has also been used in some studies (Lin and Chen 2006; Farsadnia et al. 2014). In addition, alternative classification schemes of similar num- 
ber of groups can also be evaluated, according to corresponding measures such as the Rand Index (Prinzio et al. 2011), the silhouette coefficients (Hsu and Li 2010) or are based on entropy-derived criteria (Vesanto and Alhoniemi 2000). More detailed descriptions about clustering approaches in the application of two-layer SOMs in hydrology can be found in the studies of Farsadnia et al. (2014) and Rad and Khalili (2015).

In this study, we propose a methodological framework for the application of SOMs in gridded hydroclimatic time series, which can also be helpful to spatial implementations of SOMs in other research disciplines, (Liu et al. 2016, e.g.,). The first step is the application of different sizes of SOMs, followed by hierarchical clustering and then after some analysis for regional homogeneity the spatial dependencies of the regions are presented in the form of complex networks (Figure 1).

The first step in the classification framework is the application of the SOM algorithm. The input dimensions are relatively big (2403 points x 1020 years) and the variations and characteristics of regional scPDSI values do not fluctuate quickly or greatly at the spatial scale, as they exhibit strong spatial cross-correlation patterns (Cook et al. 2015). In such cases, it has been shown that a relative small number of nodes of orthogonal structure can be efficiently implemented (Chang et al. 2014). Therefore, we used three structures of $6 \times 6,10 \times 10$ and $20 \times 20$ nodes, to examine the effect of node size in the classification process. This range of size is also in agreement with the two-layer framework proposed by (Vesanto and Alhoniemi 2000), because the set of nodes (36, 100 or 400 respectively) is much larger than the expected number of clusters that will represent the regions with similar hydroclimatic variability. The three SOMs were created by 10,000 iterations over a hexagonal grid. In the case of gridded data, each grid cell was attributed to a single node of the output layer (unified-distance matrix or U-Matrix), according to its Euclidian distance (for details in the application of SOM algorithm, see Wehrens and Kruisselbrink (2018)). Then, we utilized the agglomerative clustering method to create the second layer and determine the homogeneous 
regions as suggested by Kaufman and Rousseeuw (1990) and elaborated in Murtagh and Legendre (2014). We shall call this second step 'classification scheme'.

Instead of estimating an optimum number of clusters with one of the above-mentioned methods, e.g., C-index, we explore the within-cluster homogeneity and between-clusters heterogeneity of each different classification scheme from 2 to 30 clusters. To examine the homogeneity within each cluster, we estimate the mean of the standard deviations of scPDSI values per year per cluster; a straightforward, intuitive method to measure variability. At the same time, we measure the heterogeneity among clusters using the cross-correlation matrix of the annual mean scPDSI time series per cluster. Here, we determine the mean of the maximum cross-correlation coefficient of each cluster with the rest time-series. As the total number of clusters increase the within-cluster standard deviation will increase, while the cross-correlation between the neighbouring clusters, i.e., maximum, will decline.

Lastly, the dependence structure of the resulting clusters were then explored with the complex network method. The data representation in complex networks permits us to unify the structural complexity and vertex and connection diversities. Since graph theory (Bollobás 1998; West 2000) is the natural framework for the exact mathematical treatment of complex networks and, formally, a complex network can be represented as a graph. The algorithm used in our case to construct scalefree complex network is based on Newman and Girvan (2004) and described and applied in Tsonis et al. (2011). Firstly, a link as defined by the correlation threshold (in our case 0.5) is considered an edge connecting two clusters (nodes). Once the edges in a network have been defined we then proceed with identifying the communities.

The classification framework was developed in R statistical software and the SOM algorithm was developed by Wehrens and Kruisselbrink (2018) in kohonen package. The spatial SOM methodology presented here, was also developed as a stand-alone package, namely somspace, 
which is freely available and can be downloaded through CRAN server or alternatively at https://github.com/imarkonis/somspace.

\section{Results \& Discussion}

The comparison of the homogeneity within the final clusters derived from different node sets $(6 \times 6,10 \times 10$ and 20x20), suggests there is no strong dependency between the number of nodes and the resulting classification scheme (Figure 2). There are some small deviations between each node set, but this is expected due to the iterative nature of the algorithm, which introduces a certain amount of uncertainty in our results. Even though the uncertainty quantification lies beyond the scope of this study, it appears that there is no qualitative difference in the classification results. To detect the most representative number of clusters, we highlight changes in the slope of the regression curve of cluster number versus standard deviation or maximum correlation.

Taking into account these two measures, we can argue that segmentation above 10 clusters does not substantially improve the within-cluster homogeneity or between-clusters heterogeneity. This offers some insight on the maximum scale of hydroclimatic variability, which ranges from approximately $3 \times 10^{4}$ to $1.3 \times 10^{6} \mathrm{~km}^{2}$ (median $6.5 \times 10^{4} \mathrm{~km}^{2}$ ). The only small divergence can be seen in cross-correlation, where the $6 \times 6$ node set slightly outperforms the other two in $5-12$ clusters. This is also depicted in the regions clusters represent (Figure 3). There are some minor disagreements, e.g., central Italy in the 10x10 scheme share cluster with Iberian Peninsula instead of Western Balkans, but the overall picture remains unaffected by the number of nodes. Therefore, we can use the $6 x 6$ node set which has been found to perform slightly more consistently, in terms of cluster heterogeneity.

The segmentation, presented in Figure 4, is in good agreement with the corresponding results of Empirical Orthogonal Function (EOF) analysis of the same data set (Markonis et al. 2018a). 
However in the case of SOMs, the clusters are not overlapping as in EOFs, and additionally this approach shows enhanced flexibility; depending on the desirable scale of the application, the appropriate number of clusters can be chosen. In our case, since we are interested in a general description of Europe's hydroclimate, we chose the 10-cluster classification of the 6x6 SOM. The 10-cluster classification retains similar cross-correlation values with the corresponding 8/9-cluster classifications (Figure 2b), but each cluster is more homogeneous since its average standard deviation is lower (Figure 2a). All of the above advocates that the 10-cluster classification version could be a more consistent descriptor of European hydroclimate.

The first classification splits Europe zonally into a north and a south region. This is quite similar to the IPCC distinction (see for example Hanel et al. (2018)), as well as the findings of other studies supporting the different hydroclimatic behaviour between the northern Europe and the Mediterranean (Beniston et al. 1997; Stagge et al. 2017; Barcikowska et al. 2018). This pattern is linked mostly with the atmospheric circulation, and the dominating modes of North Atlantic Oscillation (NAO) in specific, which determine the cyclonic tracks over Europe and are known to create meridional dipoles such as the Iberian drying versus Scandinavian wetting (Beniston et al. 1997). The succeeding classifications follow the meridional direction, dividing both northern and southern domain into smaller partitions.

Interestingly, the hydroclimate of the region composed of British Isles, France and Germany has been identified to be affected most by NAO and/or Atlantic Multidecadal Oscillation (AMO) (Markonis et al. 2018a). A positive NAO phase favors more rainfall in these regions, whereas, when a positive NAO phase is coupled with a positive Arctic Oscillation (AO) phase then dry conditions prevail in Central and Eastern Europe due to the deepening of the polar vortex in combination with above normal heights over much of Southeastern Europe (Cavazos 2000). In our analysis, the region composed of British Isles, France and Germany remains undivided for a rela- 
tively big number of classifications, i.e., clusters 3-10 in Figure 4, to be split into two sub-regions only once for the rest of classification schemes (11-17).

Circulation in the common era

Another noteworthy feature includes the Mediterranean Eastern-Western segmentation, as depicted in the meridional divisions in classification schemes of $4,6,14$ clusters. This is related to the Mediterranean wet season precipitation dipole (Kutiel et al. 1996), associated with the influence of the subtropical high that emerges over in the Iberian Peninsula and northwestern Africa in conjunction with a persisting trough stretching from Greenland over Central Europe to the northeastern coast of Africa (Xoplaki et al. 2004; Roberts et al. 2012). The subtropical high is connected with subsidence, stable atmospheric conditions and thus reduced changes of precipitation, while in southeastern part of the trough there is enhanced vorticity and thus there is atmospheric instability, strong uplift, condensation and increased precipitation (Xoplaki et al. 2004).

There is evidence that this climatic pattern has been operating in the Mediterranean since the beginning of the previous millennium (Roberts et al. 2012), although this hypothesis has been recently challenged (Cook et al. 2016).

At the higher latitudes, Sweden and Norway form a single persisting cluster for most of the classification schemes (5-17). In the palaeoclimatic study of Drobyshev et al. (2016), it has been suggested that the Atlantic Sea Surface Temperatures are associated with cold conditions which direct precipitation southwards and thus are linked with dry conditions over Sweden. The influence of Atlantic Ocean, Atlantic meridional overturning thermohaline circulation (AMOC) was confirmed for the last 50 years by Ols et al. (2018), correlated with NAO and Arctic Oscillation (AO). According to their study, the sign and strength of the atmospheric indices correlation depends on the season, with the most significant correlation appearing for positive summer AMOC, NAO and $\mathrm{AO}$, which favors more precipitation. However, it was also highlighted the seasonal component 
of the association, as well as the multi-decadal shifts in correlation patterns. Similar results are presented by (Seftigen et al. 2017), highlighting strong correlations between atmospheric pressure patterns and the Sweden/Norway hydroclimate found in both observational records and simulation results.

Finally, a persisting narrow strip is detected (clusters 5-15), which extends from Pyrenees over Alps, and ends at Czech Republic. The link between Pyrenees, southern France and Alps is in good agreement with the findings of Büntgen et al. (2017). Our results suggest, though, that the correlation pattern penetrates further into central Europe. This hydroclimatic feature is identified for first time and further research is needed to rule out the possibility of spurious dependencies due to the regression bias in the development of the original gridded dataset (OWDA) or to erroneous classifications in the SOM. However, the complex network analysis suggests that this region is correlated with its the North-Western and South-Eastern neighbours and interestingly not with the Iberian Peninsula (Figure 5).

This zonally-modulated behaviour can be found at other nodes of the complex network, such as Scandinavia or Northern Italy/Western Balkans regions, providing some insight on the general behaviour of hydroclimatic variability over Europe. Two major branches are evident, both emerging from the British/France/Germany region. The first one links high latitude regions, while the other propagates over the southern ones. Since the originating region has been found to be linked with the atmospheric/oceanic circulation (Markonis et al. 2018a), we can speculate that these two branches represent the effect of the large-scale drivers to European hydroclimate. It should be underlined though, that the Iberian Peninsula appears not to be linked with the other regions. In fact, there is some weak anti-correlation with Scandinavia, as expected due to the NAO effect (Hurrell 1995) and some weak correlation with Northern Italy (Figure 5; cross-correlation matrix). 
The dependencies and differences between the mean scPDSI of each region are finally presented by visualizing each time series in Figure 6 . Some substantial negative deviations from the mean hydroclimatic conditions can be seen in the majority of the regions, corresponding to the European multi-decadal dry periods proposed by Markonis et al. (2018a). However, after the SOM classification the differences in synchronicity and sensitivity of the events can be seen, reflecting the cross-correlation matrix of Figure 5. Interestingly, this major events appear to affect mostly the western, northern and central part of Europe further supporting the role of atmospheric/oceanic circulation to multi-decadal conditions. In terms of single-year drought events, the classification appears to be in good agreement with the spatial extent of the extreme droughts of 1921 and 1976 (Moravec et al. 2019 and references within) as reconstructed by Hanel et al. (2018).

Droughts of the past

\section{Conclusions}

In this study, we applied the SOMs classification technique to a state-of-the-art, gridded, palaeoclimatic dataset in order to detect homogeneous regions of hydroclimatic variability and explored the spatial associations between them. We implemented two easily interpretive measures of withincluster homogeneity and between-cluster heterogeneity and further applied complex networks to the SOMs results. The conclusions not only successfully confirm the similar hydroclimatic behaviour of regions linked with known climatic processes, e.g., British Isles, France, Germany and NAO, but also pinpoint a region of hydroclimatic homogeneity that as far as we know has not been reported until now (Pyrenees to Czech Republic). It remains to be seen if future research will support this evidence and provide a satisfactory explanation, or it is some artifact related to the uncertainty in SOMs iterative procedure. 
The study of the inter-dependencies with complex networks highlights two diverging branches of hydroclimatic variability. They both begin at the western coasts of France and Germany and extend northwards and southwards correspondingly. The most plausible explanation stems from the large-scale drivers of hydroclimate, i.e., atmospheric and oceanic circulation. Another finding is that Iberian Peninsula is not found to be so strongly interconnected to the rest of Europe, which should be further investigated. Future research should also focus in the study of the temporal component of the cluster and network structures. For example, Ols et al. (2018) have found that the influence of oceanic circulation to Scandinavian hydroclimatic conditions is shifting over a decadal scale. Thus, it would be beneficial to see how the regions and their cross-correlation change in time.

The methodology presented can be used efficiently for exploratory spatial data analysis, reducing the system features to the most representative ones and thus allowing for easier further analysis and interpretation. Its application here resulted to a presentation of the backbone of European hydroclimatic variability, acting also as a potential indicator of the associated climatic processes. The former can lead to improvements in large-scale hydrological modelling through better discrimination of the homogeneous areas in various spatial scales, while the later could be useful in the comprehension of the complex links between climate and hydrology.

Acknowledgments. This study was funded by the Czech Science Foundation (grant no. 9-24089J) and Internal Grant Agency of The Faculty of Environmental Sciences (grant no. 20164230).

\section{References}

Baek, S. H., J. E. Smerdon, S. Coats, A. P. Williams, B. I. Cook, E. R. Cook, and R. Seager, 2017: Precipitation, temperature, and teleconnection signals across the combined north american, monsoon asia, and old world drought atlases. Journal of Climate, (2017). 
Barcikowska, M. J., S. B. Kapnick, and F. Feser, 2018: Impact of large-scale circulation changes in the north atlantic sector on the current and future mediterranean winter hydroclimate. Climate Dynamics, 50 (5-6), 2039-2059.

Beniston, M., H. Diaz, and R. Bradley, 1997: Climatic change at high elevation sites: an overview. Climatic Change, 36 (3-4), 233-251.

Benito, G., and Coauthors, 2004: Use of systematic, palaeoflood and historical data for the improvement of flood risk estimation. Review of scientific methods. Natural hazards, 31 (3), 623643.

Bollobás, B., 1998: Random graphs. Modern graph theory, Springer, 215-252.

Büntgen, U., and Coauthors, 2017: New tree-ring evidence from the pyrenees reveals western mediterranean climate variability since medieval times. Journal of Climate, 30 (14), 5295-5318.

Caillouet, L., J.-P. Vidal, E. Sauquet, A. Devers, and B. Graff, 2017: Ensemble reconstruction of spatio-temporal extreme low-flow events in France since 1871. Hydrology and Earth System Sciences, 21 (6), 2923-2951, doi:10.5194/hess-21-2923-2017.

Caliński, T., and J. Harabasz, 1974: A dendrite method for cluster analysis. Communications in Statistics-theory and Methods, 3 (1), 1-27.

Carrer, M., and C. Urbinati, 2001: Assessing climate-growth relationships: a comparative study between linear and non-linear methods. Dendrochronologia, 19 (1), 57-65.

Carro-Calvo, L., S. Salcedo-Sanz, and J. Luterbacher, 2013: Neural computation in paleoclimatology: General methodology and a case study. Neurocomputing, 113, 262-268.

Cavazos, T., 2000: Using self-organizing maps to investigate extreme climate events: An application to wintertime precipitation in the balkans. Journal of climate, $\mathbf{1 3}$ (10), 1718-1732. 
Cavazos, T., A. C. Comrie, and D. M. Liverman, 2002: Intraseasonal variability associated with wet monsoons in southeast arizona. Journal of Climate, 15 (17), 2477-2490.

Chang, F.-J., L.-C. Chang, H.-S. Kao, and G.-R. Wu, 2010: Assessing the effort of meteorological variables for evaporation estimation by self-organizing map neural network. Journal of Hydrology, 384 (1), 118-129.

Chang, L.-C., H.-Y. Shen, and F.-J. Chang, 2014: Regional flood inundation nowcast using hybrid som and dynamic neural networks. Journal of Hydrology, 519, 476-489.

Cook, B. I., K. J. Anchukaitis, R. Touchan, D. M. Meko, and E. R. Cook, 2016: Spatiotemporal drought variability in the mediterranean over the last 900 years. Journal of Geophysical Research: Atmospheres.

Cook, E. R., K. J. Anchukaitis, B. M. Buckley, R. D. DArrigo, G. C. Jacoby, and W. E. Wright, 2010: Asian monsoon failure and megadrought during the last millennium. Science, 328 (5977), $486-489$.

Cook, E. R., C. A. Woodhouse, C. M. Eakin, D. M. Meko, and D. W. Stahle, 2004: Long-term aridity changes in the western United States. Science, 306 (5698), 1015-1018.

Cook, E. R., and Coauthors, 2015: Old World megadroughts and pluvials during the Common Era. Science advances, 1 (10), e1500 561.

Cortese, G., J. K. Dolven, K. R. Bjørklund, and B. A. Malmgren, 2005: Late pleistocene-holocene radiolarian paleotemperatures in the norwegian sea based on artificial neural networks. Palaeogeography, Palaeoclimatology, Palaeoecology, 224 (4), 311-332.

Davies, D. L., and D. W. Bouldin, 1979: A cluster separation measure. IEEE transactions on pattern analysis and machine intelligence, (2), 224-227. 
D'Odorico, P., R. Revelli, and L. Ridolfi, 2000: On the use of neural networks for dendroclimatic reconstructions. Geophysical Research Letters, 27 (6), 791-794.

Drobyshev, I., Y. Bergeron, A. De Vernal, A. Moberg, A. A. Ali, and M. Niklasson, 2016: Atlantic ssts control regime shifts in forest fire activity of northern scandinavia. Scientific reports, $\mathbf{6}$, 22532.

Edwards, T. W., and Coauthors, 2017: Seasonal variability in northern hemisphere atmospheric circulation during the medieval climate anomaly and the little ice age. Quaternary Science Reviews, 165, 102-110.

Farsadnia, F., M. R. Kamrood, A. M. Nia, R. Modarres, M. Bray, D. Han, and J. Sadatinejad, 2014: Identification of homogeneous regions for regionalization of watersheds by two-level self-organizing feature maps. Journal of Hydrology, 509, 387-397.

Giraudel, J., D. Aurelle, P. Berrebi, and S. Lek, 2000: Application of the self-organizing mapping and fuzzy clustering to microsatellite data: how to detect genetic structure in brown trout (salmo trutta) populations. Artificial Neuronal Networks, Springer, 187-202.

Grieger, B., 2002: Interpolating paleovegetation data with an artificial neural network approach. Global and Planetary Change, 34 (3-4), 199-208.

Guiot, J., A. Nicault, C. Rathgeber, J. Edouard, F. Guibal, G. Pichard, and C. Till, 2005: Last-millennium summer-temperature variations in western europe based on proxy data. The Holocene, 15 (4), 489-500.

Hanel, M., O. Rakovec, Y. Markonis, P. Máca, L. Samaniego, J. Kyselỳ, and R. Kumar, 2018: Revisiting the recent european droughts from a long-term perspective. Scientific Reports, 8 (1), 9499. 
Hentati, A., A. Kawamura, H. Amaguchi, and Y. Iseri, 2010: Evaluation of sedimentation vulnerability at small hillside reservoirs in the semi-arid region of tunisia using the self-organizing map. Geomorphology, 122 (1-2), 56-64.

Ho, M., A. S. Kiem, and D. C. Verdon-Kidd, 2015a: A paleoclimate rainfall reconstruction in the Murray-Darling Basin (MDB), Australia: 1. Evaluation of different paleoclimate archives, rainfall networks, and reconstruction techniques. Water Resour. Res., 51 (10), 8362-8379, doi: 10.1002/2015WR017058.

Ho, M., A. S. Kiem, and D. C. Verdon-Kidd, 2015b: A paleoclimate rainfall reconstruction in the Murray-Darling Basin (MDB), Australia: 2. Assessing hydroclimatic risk using paleoclimate records of wet and dry epochs. Water Resour. Res., 51 (10), 8380-8396.

Ho, M., U. Lall, and E. R. Cook, 2016: Can a paleodrought record be used to reconstruct streamflow?: A case study for the Missouri River Basin. Water Resour. Res., 52 (7), 5195-5212, doi:10.1002/2015WR018444.

Ho, M., U. Lall, X. Sun, and E. R. Cook, 2017: Multiscale temporal variability and regional patterns in 555 years of conterminous U.S. streamflow. Water Resources Research, doi:10.1002/ 2016WR019632.

Hosking, J., and J. Wallis, 1997: Regional frequency analysis, 224 pp. Cambridge University.

Hsu, K.-C., and S.-T. Li, 2010: Clustering spatial-temporal precipitation data using wavelet transform and self-organizing map neural network. Advances in Water Resources, 33 (2), 190-200.

Hubert, L., and J. Schultz, 1976: Quadratic assignment as a general data analysis strategy. British journal of mathematical and statistical psychology, 29 (2), 190-241. 
Hurrell, J. W., 1995: Decadal trends in the north atlantic oscillation: regional temperatures and precipitation. Science, 269 (5224), 676-679.

Juggins, S., G. L. Simpson, and R. J. Telford, 2015: Taxon selection using statistical learning techniques to improve transfer function prediction. The Holocene, 25 (1), 130-136.

Kalteh, A. M., P. Hjorth, and R. Berndtsson, 2008: Review of the self-organizing map (som) approach in water resources: Analysis, modelling and application. Environmental Modelling \& Software, 23 (7), 835-845.

Kaufman, L., and P. Rousseeuw, 1990: Finding groups in data, 1990. New York, 22-52.

Keller, T., J. Guiot, and L. Tessier, 1997: Climatic effect of atmospheric co2 doubling on radial tree growth in south eastern france. Journal of Biogeography, 24 (6), 857-864.

Kohonen, T., 2001: Self-organizing maps, ser. Information Sciences. Berlin: Springer, 30.

Kutiel, H., P. Maheras, and S. Guika, 1996: Circulation and extreme rainfall conditions in the eastern mediterranean during the last century. International Journal of Climatology, 16 (1), 73 92.

Lary, D. J., A. H. Alavi, A. H. Gandomi, and A. L. Walker, 2016: Machine learning in geosciences and remote sensing. Geoscience Frontiers, 7 (1), 3-10, doi:10.1016/j.gsf.2015.07.003, URL http://www.sciencedirect.com/science/article/pii/S1674987115000821.

Ley, R., M. Casper, H. Hellebrand, and R. Merz, 2011: Catchment classification by runoff behaviour with self-organizing maps (som). Hydrology and Earth System Sciences, 15 (9), 2947.

Lin, G.-F., and L.-H. Chen, 2006: Identification of homogeneous regions for regional frequency analysis using the self-organizing map. Journal of Hydrology, 324 (1), 1-9. 
Liu, Y., R. H. Weisberg, S. Vignudelli, and G. T. Mitchum, 2016: Patterns of the loop current system and regions of sea surface height variability in the eastern Gulf of Mexico revealed by the self-organizing maps. Journal of Geophysical Research: Oceans, 121 (4), 2347-2366, URL http://onlinelibrary.wiley.com/doi/10.1002/2015JC011493/full.

Malmgren, B. A., and U. Nordlund, 1997: Application of artificial neural networks to paleoceanographic data. Palaeogeography, Palaeoclimatology, Palaeoecology, 136 (1-4), 359-373.

Markonis, Y., M. Hanel, P. Máca, J. Kyselỳ, and E. Cook, 2018a: Persistent multi-scale fluctuations shift european hydroclimate to its millennial boundaries. Nature communications, 9 (1), 1767.

Markonis, Y., Y. Moustakis, C. Nasika, P. Sychova, P. Dimitriadis, M. Hanel, P. Máca, and S. Papalexiou, 2018b: Global estimation of long-term persistence in annual river runoff. Advances in Water Resources, 113, 1-12.

Moravec, V., Y. Markonis, O. Rakovec, R. Kumar, and M. Hanel, 2019: A 250-year european drought inventory derived from ensemble hydrologic modelling. Geophysical Research Letters, doi:10.1029/2019GL082783.

Murtagh, F., and P. Legendre, 2014: Wards hierarchical agglomerative clustering method: which algorithms implement wards criterion? Journal of Classification, 31 (3), 274-295.

Newman, M. E., and M. Girvan, 2004: Finding and evaluating community structure in networks. Physical review E, 69 (2), 026113.

Ni, F., T. Cavazos, M. K. Hughes, A. C. Comrie, and G. Funkhouser, 2002: Cool-season precipitation in the southwestern usa since ad 1000: comparison of linear and nonlinear techniques for 
reconstruction. International Journal of Climatology: A Journal of the Royal Meteorological Society, 22 (13), 1645-1662.

Nourani, V., A. H. Baghanam, J. Adamowski, and M. Gebremichael, 2013: Using self-organizing maps and wavelet transforms for space-time pre-processing of satellite precipitation and runoff data in neural network based rainfall-runoff modeling. Journal of Hydrology, 476, 228-243.

Ols, C., V. Trouet, M. P. Girardin, A. Hofgaard, Y. Bergeron, and I. Drobyshev, 2018: Post-1980 shifts in the sensitivity of boreal tree growth to north atlantic ocean dynamics and seasonal climate: Tree growth responses to north atlantic ocean dynamics. Global and Planetary Change, $165,1-12$.

Papacharalampous, G., H. Tyralis, and D. Koutsoyiannis, 2017: Forecasting of geophysical processes using stochastic and machine learning algorithms.

Pérez-Ortiz, M., A. M. Durán-Rosal，P. A. Gutiérrez, J. Sánchez-Monedero, A. Nikolaou, F. Fernández-Navarro, and C. Hervás-Martínez, 2019: On the use of evolutionary time series analysis for segmenting paleoclimate data. Neurocomputing, 326, 3-14.

Peyron, O., and A. d. Vernal, 2001: Application of artificial neural networks (ann) to high-latitude dinocyst assemblages for the reconstruction of past sea-surface conditions in arctic and subarctic seas. Journal of Quaternary Science: Published for the Quaternary Research Association, 16 (7), 699-709.

Pozzi, M., B. A. Malmgren, and S. Monechi, 2000: Sea surface-water temperature and isotopic reconstructions from nannoplankton data using artificial neural networks. Palaeontologia Electronica, 3 (2), 1-14. 
Prinzio, M. D., A. Castellarin, and E. Toth, 2011: Data-driven catchment classification: application to the pub problem. Hydrology and Earth System Sciences, 15 (6), 1921-1935.

Punyasena, S. W., D. K. Tcheng, C. Wesseln, and P. G. Mueller, 2012: Classifying black and white spruce pollen using layered machine learning. New Phytologist, 196 (3), 937-944.

Rad, A. M., and D. Khalili, 2015: Appropriateness of clustered raingauge stations for spatiotemporal meteorological drought applications. Water resources management, 29 (11), 4157.

Reusch, D. B., 2010: Nonlinear climatology and paleoclimatology: capturing patterns of variability and change with self-organizing maps. Physics and Chemistry of the Earth, Parts $A / B / C$, 35 (9-12), 329-340.

Roberts, N., and Coauthors, 2012: Palaeolimnological evidence for an east-west climate see-saw in the mediterranean since ad 900. Global and Planetary Change, 84, 23-34.

Rodríguez-Alarcón, R., and S. Lozano, 2017: Som-based decision support system for reservoir operation management. Journal of Hydrologic Engineering, 22 (7), 04017012.

Rousi, E., U. Ulbrich, H. W. Rust, and C. Anagnostolpoulou, 2017: An NAO Climatology in Reanalysis Data with the Use of Self-organizing Maps. Perspectives on Atmospheric Sciences, Springer, Cham, 719-724, dOI: 10.1007/978-3-319-35095-0_103.

Salonen, J. S., M. Luoto, T. Alenius, M. Heikkilä, H. Seppä, R. J. Telford, and H. J. B. Birks, 2014: Reconstructing palaeoclimatic variables from fossil pollen using boosted regression trees: comparison and synthesis with other quantitative reconstruction methods. Quaternary Science Reviews, 88, 69-81.

Schook, D. M., J. M. Friedman, and S. L. Rathburn, 2016: Flow reconstructions in the Upper Missouri River Basin using riparian tree rings. Water Resources Research, 52 (10), 8159-8173. 
Seftigen, K., H. Goosse, F. Klein, and D. Chen, 2017: Hydroclimate variability in scandinavia over the last millennium-insights from a climate model-proxy data comparison. Climate of the Past, 13, 1831.

Spraggs, G., L. Peaver, P. Jones, and P. Ede, 2015: Re-construction of historic drought in the Anglian Region (UK) over the period 1798-2010 and the implications for water resources and drought management. Journal of Hydrology, 526, 231-252.

Srinivas, V., S. Tripathi, A. R. Rao, and R. S. Govindaraju, 2008: Regional flood frequency analysis by combining self-organizing feature map and fuzzy clustering. Journal of Hydrology, 348 (1-2), 148-166.

Stagge, J. H., D. G. Kingston, L. M. Tallaksen, and D. M. Hannah, 2017: Observed drought indices show increasing divergence across europe. Scientific Reports, 7 (1), 14045.

Steinschneider, S., M. Ho, E. R. Cook, and U. Lall, 2016: Can PDSI inform extreme precipitation?: An exploration with a 500 year long paleoclimate reconstruction over the U.S. Water Resources Research, 52 (5), 3866-3880, doi:10.1002/2016WR018712.

Toth, E., 2013: Catchment classification based on characterisation of streamflow and precipitation time series. Hydrology and Earth System Sciences, 17 (3), 1149.

Tsonis, A. A., G. Wang, K. L. Swanson, F. A. Rodrigues, and L. da Fontura Costa, 2011: Community structure and dynamics in climate networks. Climate dynamics, 37 (5-6), 933-940.

Tyralis, H., G. Papacharalampous, and A. Langousis, 2019: A brief review of random forests for water scientists and practitioners and their recent history in water resources. Water, 11 (5), 910.

Ultsch, A., and H. P. Siemon, 1990: Kohonen's self organizing feature maps for exploratory data analysis. Proceedings of the International Neural Network Conference (INNC-90), Paris, 
France, July 913, 1990 1. Dordrecht, Netherlands, B. Widrow, and B. Angeniol, Eds., Kluwer Academic Press, Dordrecht, Netherlands, Vol. 1, 305-308, URL http://www.uni-marburg.de/ fb12/datenbionik/pdf/pubs/1990/UltschSiemon90.

Vesanto, J., and E. Alhoniemi, 2000: Clustering of the self-organizing map. IEEE Transactions on neural networks, 11 (3), 586-600.

Wehrens, R., and J. Kruisselbrink, 2018: Flexible self-organising maps in kohonen 3.0. Journal of Statistical Software, 87 (7).

West, D. B., 2000: Introduction to Graph Theory. 2nd ed., Prentice Hall.

Wise, E. K., and M. P. Dannenberg, 2014: Persistence of pressure patterns over north america and the north pacific since ad 1500. Nature communications, 5, 4912.

Woodhouse, C. A., 1999: Artificial neural networks and dendroclimatic reconstructions: an example from the front range, colorado, usa. The Holocene, 9 (5), 521-529.

Xoplaki, E., J. González-Rouco, J. u. Luterbacher, and H. Wanner, 2004: Wet season mediterranean precipitation variability: influence of large-scale dynamics and trends. Climate dynamics, 23 (1), 63-78. 


\section{LIST OF FIGURES}

Fig. 1. Visual summary of the classification framework. . . . . . . . . . . . . . . . . . 27

Fig. 2. Homogeneity/heterogeneity of different classification schemes. Mean cluster standard deviation (a) and mean maximum cross-correlation (b) versus number of clusters, for the three SOMs structures. Smoothed lines are produced by loess regression. . . . . . . . . . 28

Fig. 3. Classification schemes consisting of 9 clusters for $6 \times 6,10 \times 10$ and 20x20 SOM structures (left to right).

Fig. 4. Different classification schemes for 6x6 SOM structure, covering 2 to 17 clusters. $\quad . \quad$. . . 30

Fig. 5. Network analysis of the SOM clusters (10 clusters). Only nodes with cross-correlation coefficients above 0.5 are depicted in the superimposition of the complex network with the SOM cluster (left panel). The exact values can be seen in the correlation matrix on the right panel.

Fig. 6. Mean value of scPDSI for the clusters of Figure 5 (loess regression; shaded area represents the $p=0.95$ confidence interval). Solid vertical lines represent the peak of the multi-decadal dry periods according to Markonis et al. (2018a), dashed vertical lines represent the 1921 and 1976 extreme drought events presented in Moravec et al. (2019) and points are the mean value of scPDSI mean values for the given years according to the classification in this study. 
OWDA

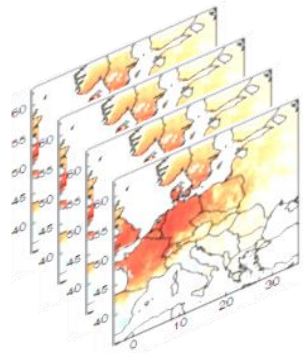

2403 grid cells $x 1020$ years

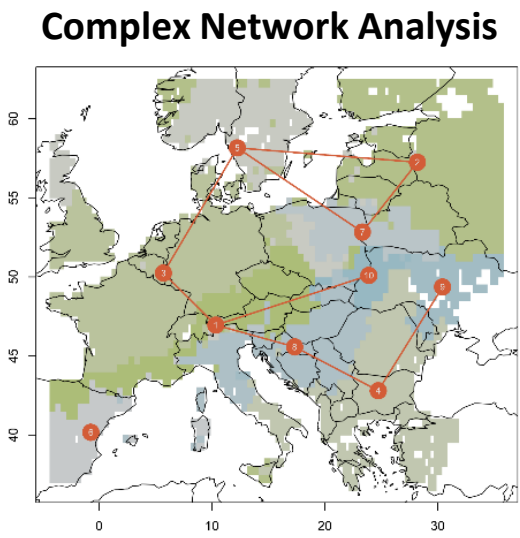

Most representative classification: 10 regions ( 6 x 6 nodes)
SOMs
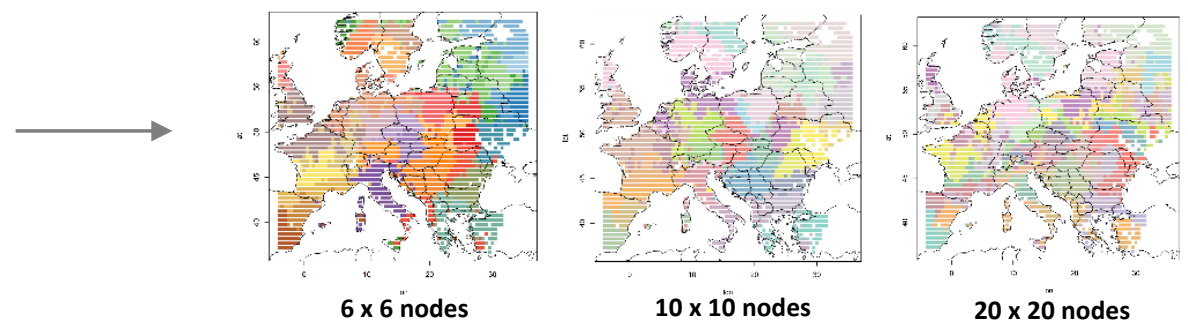

$20 \times 20$ nodes

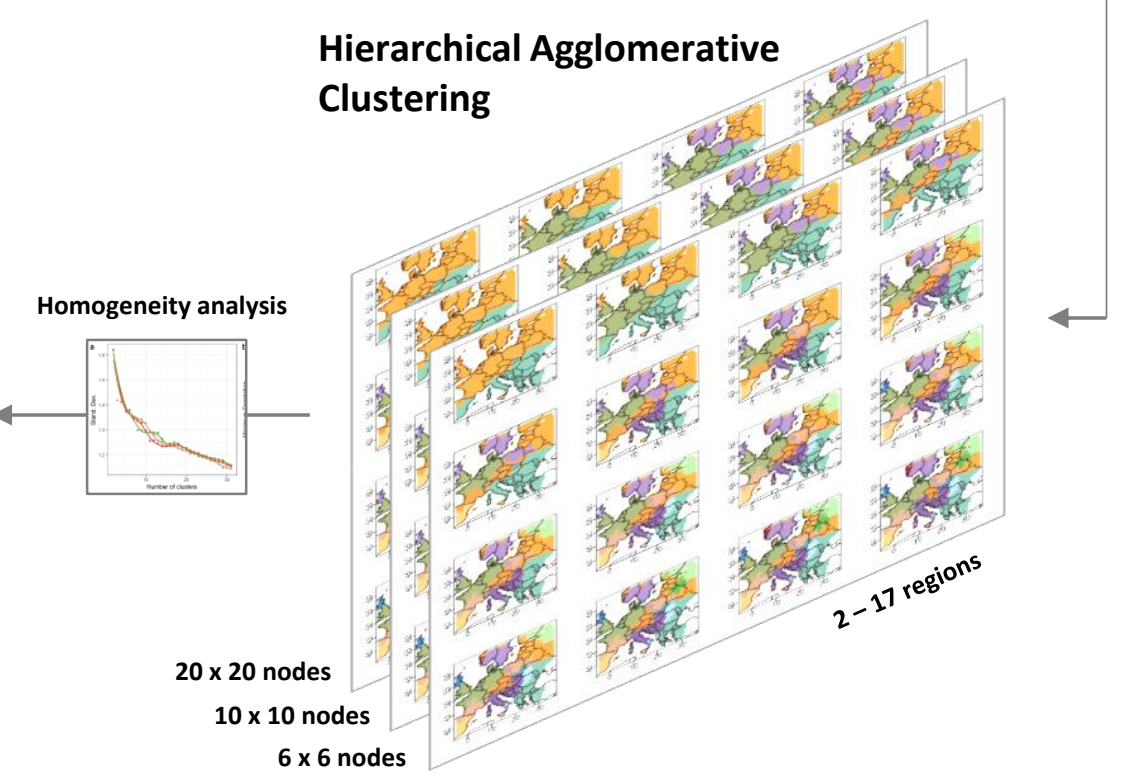

FIG. 1: Visual summary of the classification framework. 

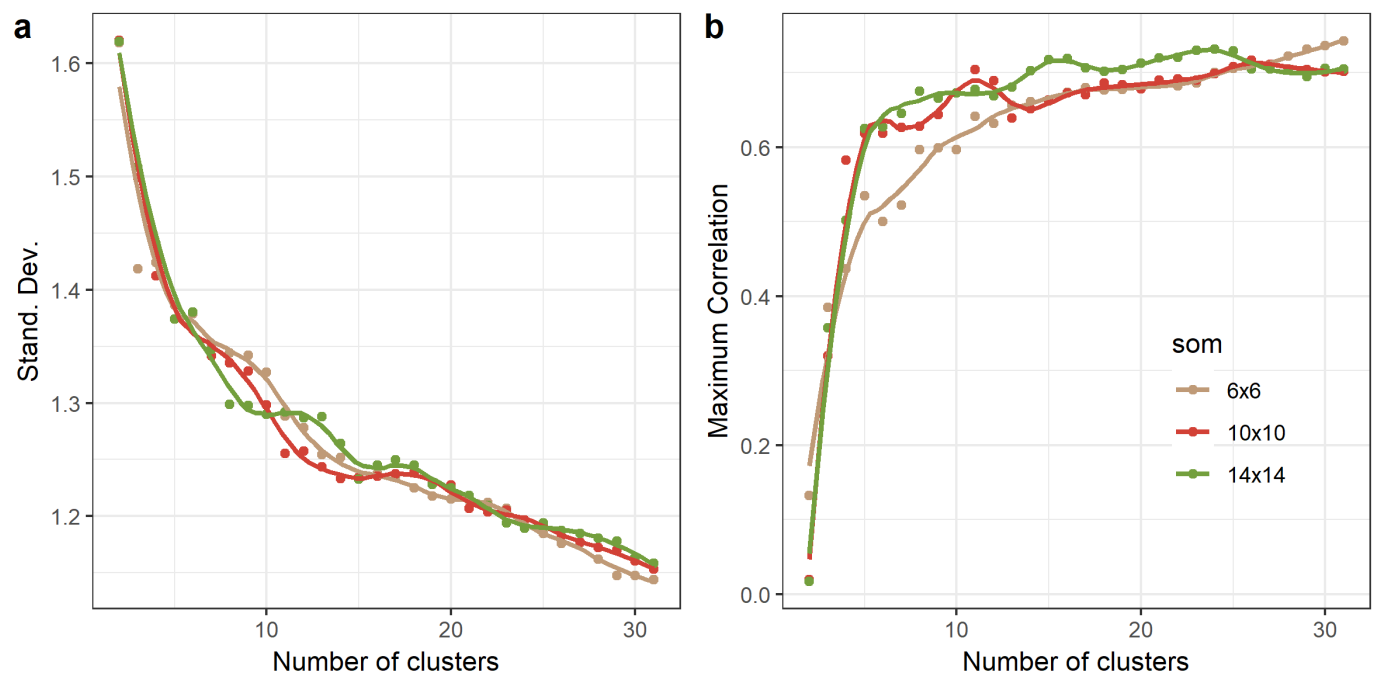

FIG. 2: Homogeneity/heterogeneity of different classification schemes. Mean cluster standard deviation (a) and mean maximum cross-correlation (b) versus number of clusters, for the three SOMs structures. Smoothed lines are produced by loess regression. 

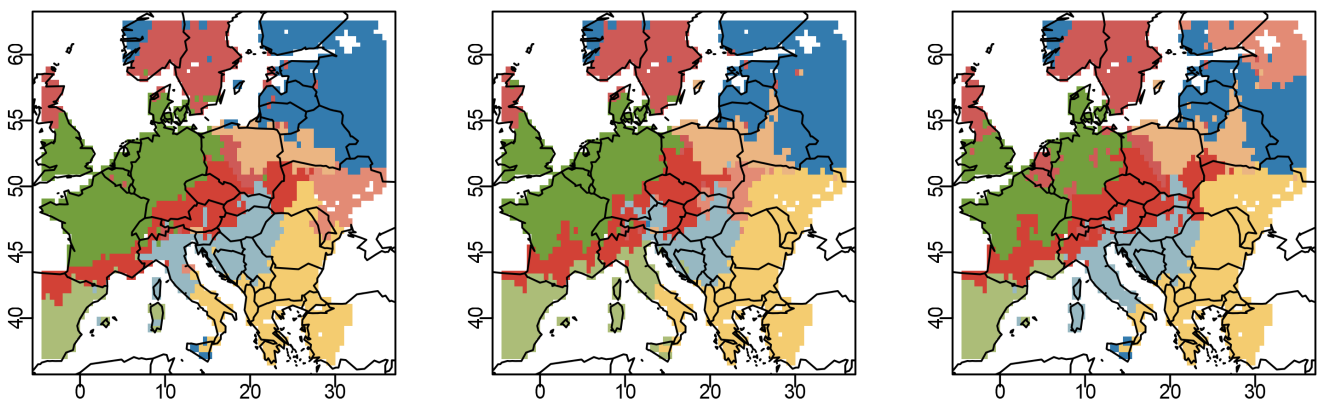

FIG. 3: Classification schemes consisting of 9 clusters for $6 \times 6,10 \times 10$ and 20x20 SOM structures (left to right). 

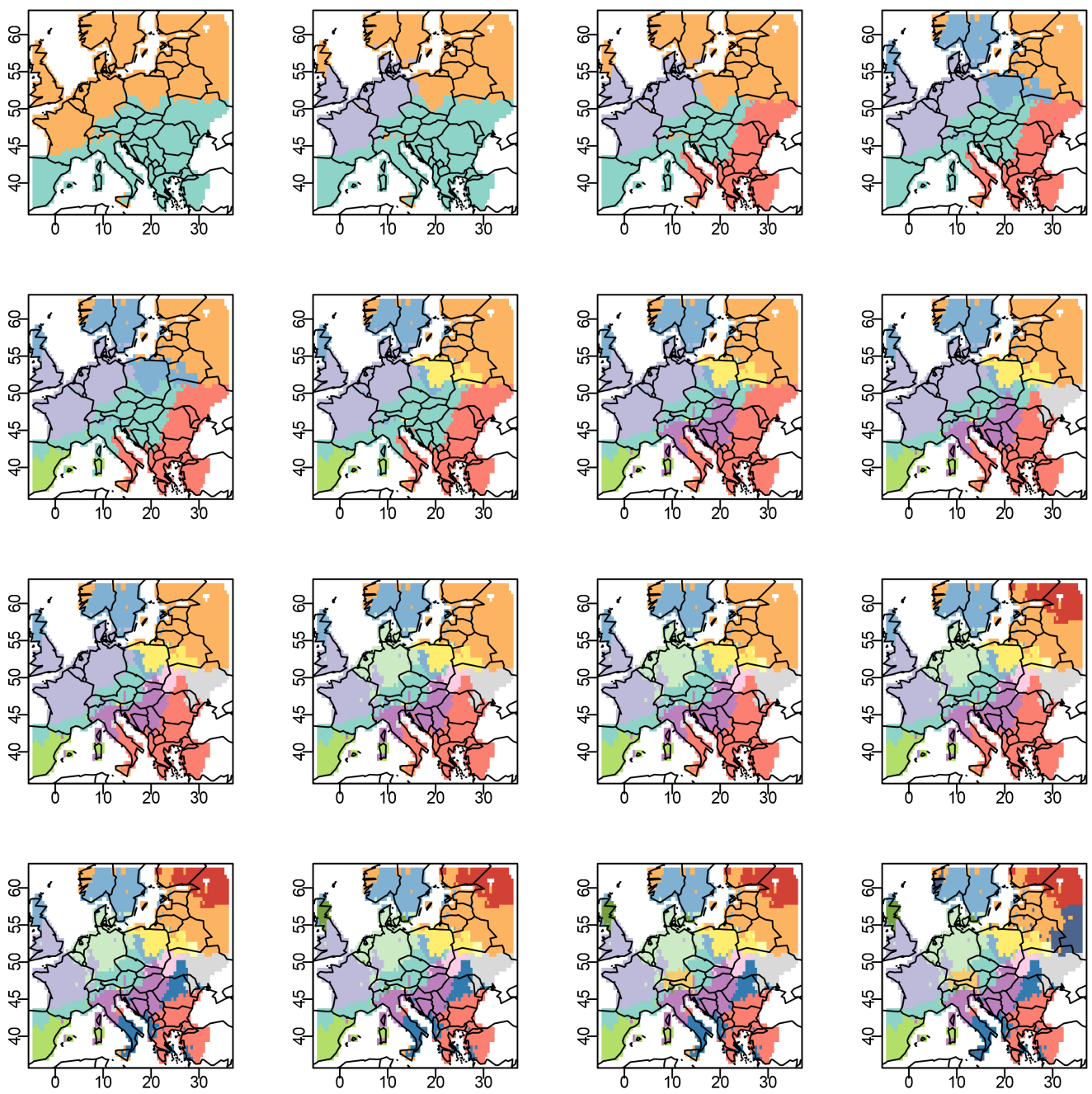

FIG. 4: Different classification schemes for 6x6 SOM structure, covering 2 to 17 clusters. 

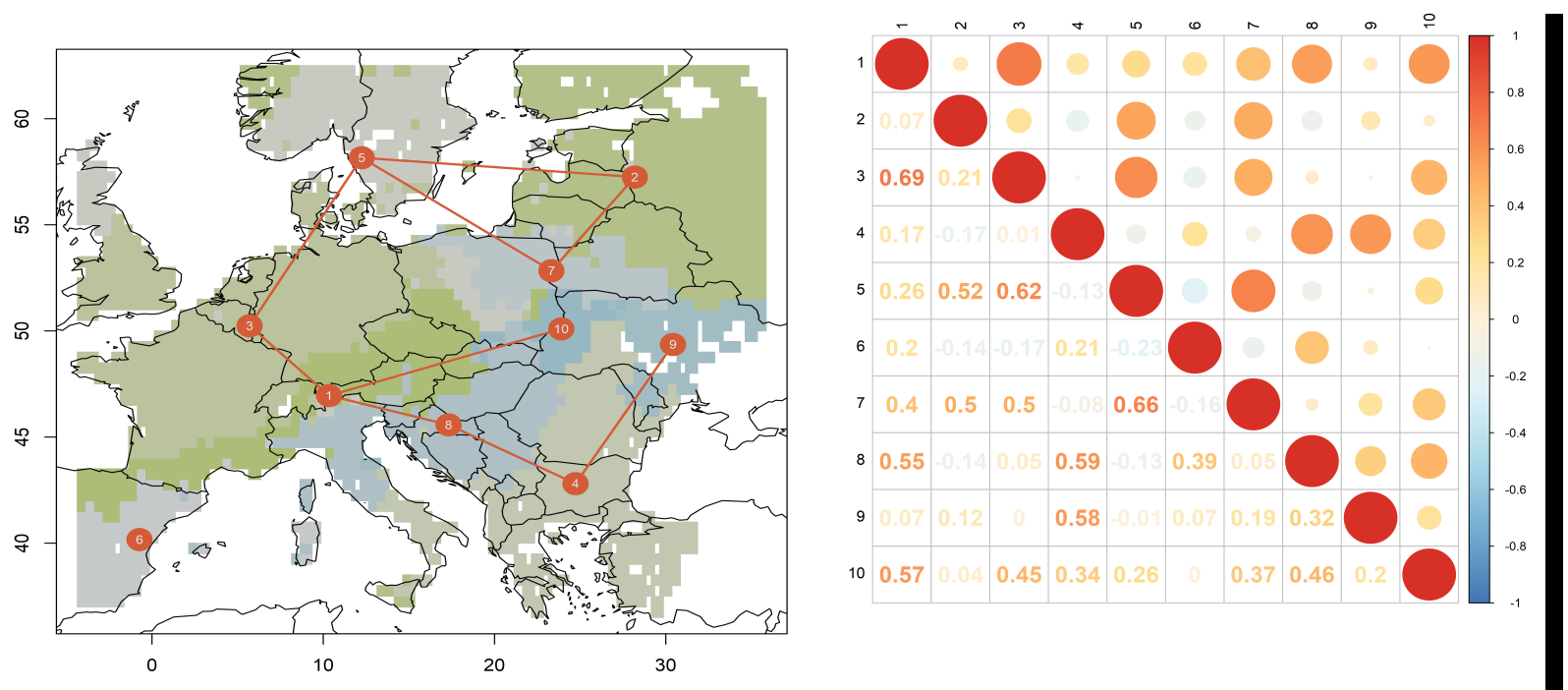

FIG. 5: Network analysis of the SOM clusters (10 clusters). Only nodes with cross-correlation coefficients above 0.5 are depicted in the superimposition of the complex network with the SOM cluster (left panel). The exact values can be seen in the correlation matrix on the right panel. 


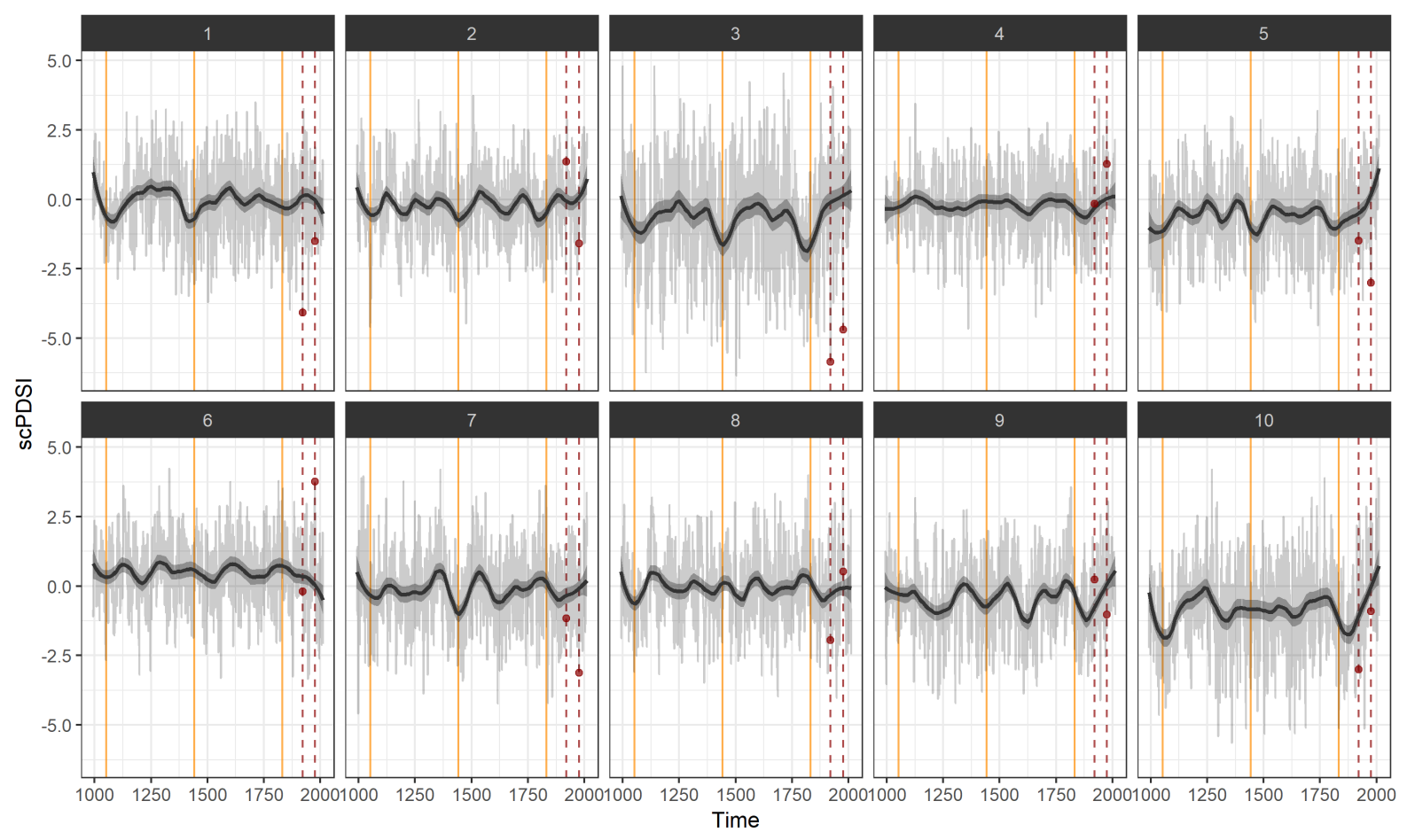

FIG. 6: Mean value of scPDSI for the clusters of Figure 5 (loess regression; shaded area represents the $p=0.95$ confidence interval). Solid vertical lines represent the peak of the multi-decadal dry periods according to Markonis et al. (2018a), dashed vertical lines represent the 1921 and 1976 extreme drought events presented in Moravec et al. (2019) and points are the mean value of scPDSI mean values for the given years according to the classification in this study. 